\title{
Índiz d'asturianidá de dos testos falaos: un ensayu
}

Recibido: 12/10/2017

Aceptado: 11/12/2017

\section{RESUMEN:}

La mezcla de asturiano y castellano plantea el problema de la frontera lectal entre ambas lenguas: ¿qué es hablar asturiano? Si se adopta un enfoque subjetivo, la respuesta depende de las percepciones del hablante. Si se adopta un enfoque objetivo, la respuesta depende de un análisis cuantitativo realizado por el lingüista. Aquí se propone un procedimiento objetivo de recuento (sin ponderación) aplicable a textos o muestras concretas de habla. Se hace el ensayo con dos textos orales, uno rural de Cabranes y otro urbano de Gijón. Los resultados son muy elocuentes sobre la tipificación de ambos textos. PALABRAS CLAVE: asturiano, castellano, mezcla de lenguas, análisis cuantitativo, rasgos diferenciales.

Asturian laguage index in spoken texts: an essay

\section{ABSTRACT:}

The mixture of Asturian and Spanish raises the problem of the lectal boundary between both languages: what is to speak Asturian? If a subjective approach is adopted, the answer depends on the perceptions of the speaker. If an objective one, the answer depends on a quantitative analysis by the linguist. Here is proposed an objective procedure count (without weighting) applicable to texts or specific samples of speech. This trial is applied to two oral texts, one rural from Cabranes and the other urban from Gijón. The results are very eloquent about the classification of both texts.

KEY WORDS: Asturian, Spanish, language mixing, quantitative analysis, differential features. 


\section{Contactu de llingües y frontera lectal}

N'Asturies produzse un contactu de llingües ente castellanu y asturianu (gallego-asturianu na so zona), tanto nel planu social como nel individual ${ }^{1}$. Como ye sabío, el contactu de llingües da pie a una serie de fenómenos d'interacción que puen resumise, fundamentalmente, nestos: interferencies o replicaciones d'un trazu llingüísticu d'un sistema nel otru; hibridación o formación de lectos híbridos (amestaos); y alternancia de códigos o emisión de cadenes falaes formaes por segmentos alternantes d'una llingua y otra ${ }^{2}$.

Dende los años 70 y 80 vien usándose «amestáu» como términu técnicu pa designar el tipu de fala híbrida que, siendo orixinariamente asturiana, integra, en mayor o menor grau según los casos, interferencies castellanes ${ }^{3}$. Fundamentalmente hai dos maneres de ver l'amestáu: como un contínuum llingüísticu indivisu y inestable ente dos polos más o menos «puros» d'asturianu y castellanu; ; o como un lectu o familia gradual de lectos discretos con della estabilidás. En concreto, n'Andrés (2002) ofrezse una hipótesis sobre la mena d'amestáu nomáu asturianu mínimu (AM) o asturianu castellanizáu d'ámbitos urbanos: taría formáu, principalmente, por un paquete compactu de

1 Les primeres alusiones directes al contactu de llingües na descripción de la situación sociollingüística d'Asturies, débense a Martínez Álvarez (1968: 8, 10).

2 Baxo esta clasificación tan xeneral esiste una fenomenoloxía más variada que nun ye oxetu d'atención nesti trabayu. Ver López Morales (2010: cap. V).

3 La visión del amestáu como una variedá contactual del asturianu ta presente en trabayos como los de Lüdtke (1999: 23), Andrés (2002: 25) o Teso (2015).

4 Esta ye la visión de Dyzmann (2000) o d'Alba Niño (2011: 222-223).

5 Con matices, ye la imaxe que se da n'Andrés (2002) o en Teso (2015). Dambes idees concíliense en Martínez Álvarez (1968: 111): «Entre los dos extremos —bable de la intimidad, castellano público y escrito - hay infinitos matices que pueden reducirse a dos tipos: bable castellanizado y castellano asturianizado». Sobre'l conceptu de contínuum llingüísticu sociolectal, ver el resume de Kabatek (1999: 216-219). 
trazos estereotípicos que faen de frontera de contención frente al castellanu'; y gociaría d'estabilidá relativa na tresmisión xeneracional. Nes descripciones de l'amestanza de llingües n'Asturies nun son rares les referencies a daqué confuso o borrinoso, onde ye imposible llantar una frontera clara ente los lectos de partida (asturianu y castellanu). A mou d'exemplu: Martínez Álvarez (1968: 8) fala d' «infinitos grados de transición»; en Cano et alii (1976: 17) testimóniase cómo los falantes d'asturianu camienten que «no hablan ni asturiano ni castellano sino una "mezcla"»; Fernández Lorences (2011: 194) observa que «[s]in tener de mano establecíu un patrón de comparación basáu tanto nos conocimientos llingüústicos diacrónicos y sincrónicos como na propia oralidá, les muestres de la llingua falada puen presentase al investigador como una realidá caótica y cambiante...»; Cuesta (2016: 148) diz que «[1]a mayor parte de las veces nos encontramos con una variedad intermedia: un castellano más o menos asturianizado, un asturiano más o menos castellanizado o una variedad dificil de definir categóricamente como una cosa o como la otra».

Pero, lóxicamente, uno ye que la fala amestada paeza daqué confuso, y otro ye que, sometida a criterios d'análisis determinaos, se compruebe nella un orde internu precisu. Hai qu'albidrar qu'una muestra de fala amestada nun será daqué onde nun podamos decidir l'adscripción a un lectu preponderante (asturianu o castellanu).

Pal propósitu mentáu, podemos remanar teóricamente dos enfoques, ún perceptivu y otru oxetivu. Acordies con un enfoque perceptivu, son los falantes los que determinen si lo que falen ye asturianu o castellanu, y polo tanto los que deciden ú la

6 Ente otros: marca axetival de masculín singular en -u; plural femenín en -es; demostrativos esti, esi; posesivu prenuclear con artículu; regles asturianes d'allugamientu de pronomes átonos col verbu; pronome átonu de complementu indirectu - $y$, -yos; pronome átonu vos; perda de $-r$ del infinitivu con pronome enclíticu; ausencia de tiempos compuestos con haber en dellos casos; formes verbales como yes, ye; etcétera. 
llende ente asturianu y castellanu, remanando como criteriu la propia percepción de los fechos llingüísticos. Esta ye, en realidá, la tesis central n'Andrés (2002): que los falantes atribuyiríen el «falar asturianu» y el «falar castellanu» a un garapiellu de trazos percibíos como estereotípicos, ensin nengún análisis oxetivu de la fala utilizada ${ }^{7}$. De xuru, l'enfoque perceptivu ye válidu y operativu, pero nun cubre más qu'una parte del problema, porque ye un criteriu sociocultural, y non glotolóxicu. Col enfoque perceptivu operamos como falantes y non como llingüistes, y asumimos qu'una muestra de fala amestada ye «asturianu»o «castellanu» basándonos namás que n’impresiones más o menos fundamentaes ${ }^{8}$.

Sicasí, acordies con un enfoque oxetivu, ye'1 llingüista'l de decidir si lo qu'emplega'l falante ye asturianu o castellanu y ú s'atopa la llende ente un lectu y otru, remanando criterios oxetivamente glotolóxicos y ayenos a suxetividaes de raigañu sociocultural ${ }^{9}$.

Por supuesto, nun se pue esperar que los resultaos del enfoque suxetivu y del oxetivu coincidan siempre. Son conocíos los

7 Nos estudios del amestáu, la percepción de los falantes tien un papel principal. Por exemplu, na encuesta de Dyzmann (2002: 99) obtiénse'l datu de qu'a los rapazos del Institutu de Roces, de Xixón, paez-yos falar un castellanu asturianizáu, ensin necesidá de determinar qué ye lo que falen «realmente».

8 Referímonos, por exemplu, a apreciaciones como esta: «Na nuestra opinión, y sin disponer de datos concluyentes, l'AM [asturianu mínimu] paez glotolóxicamente una variedá (contactual) dientro del diasistema asturianu, y ello por estes razones: porque, sincrónicamente, pesen nél más los trazos de tipu asturianu que los de tipu castellanu; y porque, diacrónicamente, surde d'una base asturiana que nun algamó un grau de castellanización talu que lu faiga cayer nel dominiu lectal castellanu» (Andrés, 2002: 33). O esta otra: «Puesto que l'amestáu ye básicamente un asturianu fuertemente castellanizáu, dependerá d'hasta ónde lleguen los elementos castellanos pa que lu consideremos un dialeutu del asturianu o del castellanu. $\mathrm{Nel}$ casu más xeneral entá güei paez más razonable entendelu como un dialeutu del asturianu»; «Si hai razones pa considerar l'amestáu más habitual como un dialeutu del asturianu, y seguramente sí les hai...» (Teso, 2015: 15, 16).

9 N'Andrés (2002: 33) llamábase l'atención sobre'1 criteriu glotolóxicu pa determinar esti problema, anque ellí nun se ponía en práctica. 
comentarios que constaten cómo'l falante «s'equivoca» camentando suxetivamente falar castellanu, cuando lo que fala oxetivamente paez ser asturianu. Por exemplu:

«Por un lado la cercanía fonológica facilita extraordinariamente el paso de un código a otro sin mayores distorsiones, hasta el punto que en muchas circunstancias puede un hablante creer que se expresa en castellano cuando en realidad lo está haciendo en bable» (Cano et alii, 1976: 18; cursiva de nuestro).

«La sensación de que [l'amestáu] ye un dialeutu del español ye pola mor de que normalmente los falantes intuyen equivocadamente que ye una variante dialeutal aquello que puen entender y que ye una llingua distinta lo que nun puen entender. Como lo que s'oye nes ciudaes asturianes, ye raro y mui chocante pal foranu, pero entiéndelo y, como l'español ye la llingua dominante, tanto'l foranu como l'asturianu sienten l'amestáu como un español con rasgos dialeutales. Por supuesto, en munchos falantes, yá mui castellanizaos, esto ye asina, pero nun creo que seya nesti momentu la norma. Partamos, entós, de que l'amestáu ye una variante dialeutal del asturianu» (Teso, 2015: 15; cursiva de nuestro).

La idea de que la resistencia de la gramática o morfosintaxis asturiana marca la sobrevivencia de la llingua frente al castellanu, pue rastrexase en diversos trabayos; por exemplu, en Martínez Álvarez (1968: 9), en Fernández Álvarez (1989), n’Andrés (2002: 25), en Viejo Fernández (2005: 142) o en Teso (2015: 15). Nun vamos entrar equí nel fundamentu teóricu de la preponderancia de la morfosintaxis; simplemente albidramos que non tolos especialistes taríen d'acuerdu con esi supuestu.

Si falamos de criterios oxetivos con cuenta de tipificar un tipu de fala, podemos tar falando guapamente de criterios cuantitativos. Ante una muestra de fala, la entruga entós ye esta: ¿ye posible entamar una cuantificación pa determinar si una muestra ta n'asturianu o en castellanu, o - en términos menos radicales - si esa muestra apila más pal asturianu o pal castellanu? ¿Y cómo se fadría una cuantificación tala? 
El sentíu común diznos qu'esa operación fadríase, simplemente, cuntando trazos propios d'un y d'otru lectu que tean presentes na muestra de fala o testu. En realidá, nun tamos falando de nenguna novedá ablucante, y menos de nengún métodu complexu. Cuando Elizaincín (1996) quixo dar la mesma respuesta referida al fronteiriço del Uruguái, ideó una «escala de variabilidá» pa midir porcentaxes tipolóxicos, pa d'esta manera decidir si una fala concreta yera «(más) española» 0 «(más) portuguesa». Nel casu del amestáu asturianu, Fernández Álvarez (1989) ensayó una cuantificación de la fala de Llue (Colunga), pero manexando namás trés trazos. Pela so parte, Prieto (1991: 21-27) tentó daqué asemeyao col amestáu de Xixón, pero aplicao namái a un pequeñu repertoriu léxicu. En dambos casos tamos ante ensayos parciales nos que non siempre paez tar claru'1 criteriu de tipificación y de selección de trazos.

\section{Índiz de proporción testual. Principios y conceptos}

Base contrastiva y términos contrastivos. Corpus contrastivu d'un testu

Vamos partir d'un supuestu elemental: un testu cualesquier (esto ye, cualesquier muestra de discursu, faláu o escritu, coloquial o ellaboráu) al que-y atribuyimos daqué hibridismu o amestanza, ta compuestu por elementos o trazos d'un lectu A y d'un lectu B, detectables y cuantificables de manera tala que se pue determinar la proporción qu'algamen unos y otros. Esti ye'l casu d'una muestra de fala producida por un habitante d'Asturies. Lo previsible ye qu'un testu d'esta mena presente trazos d'estes tipoloxíes:

1. Elementos non diferenciales: los comunes a dambes llingües.

2. Elementos diferenciales:

2a. Trazos propios del sistema asturianu: fonético-fonolóxicos, morfosintácticos, léxico-semánticos.

2b. Trazos propios del sistema castellanu: fonético-fonolóxicos, morfosintácticos, léxico-semánticos. 
Obviamente, los elementos non diferenciales nun informen un res del hibridismu, y constitúin la base común (BCom) del testu. Por exemplu, el fenómenu «forma del pronome tónicu de suxetu de $1 .{ }^{\text {er }}$ persona del singular» ye yo en dambes llingües. La BCom pue representase asina:

ast. $y o=$ cast. $y o$

Lóxicamente, l'análisis ha basase nos elementos diferenciales, qu'exercen como términos d'una base contrastiva. Llamamos base contrastiva (BC) a aquel elementu d'un testu que, siendo susceptible de presentar variación interllingüística, resuélvese na escoyeta d'ún de los términos del contraste, ye dicir, d'un trazu perteneciente a una de les llingües confrontaes, y non a la otra ${ }^{10}$. Por exemplu, nun testu (d'amestáu) asturianu ye previsible, de mano, qu'apaeza la forma pronominal ast. $-y$ o cast. le, y polo tanto la $\mathrm{BC}$ ye:

ast. $-y \|$ cast. le

Si lo qu'apaez en testu ye la forma $-y$, significa que' 1 falante escoyó'1 términu asturianu (en cursiva versal), quedando'l castellanu como opción virtual o non realizada (en cursiva non versal):

ast. $-\Upsilon \|$ cast. le

Pelo contrario, si lo qu'apaez en testu ye la forma le, significa que'l falante escoyó 1 términu castellanu (en cursiva versal), quedando l'asturianu como opción virtual (en cursiva blanco):

ast. $-y \|$ cast. LE

10 Pal conceptu de base contrastiva aplicáu al contactu d'asturianu y castellanu, ver Andrés (2002: 22), Andrés (2015) y Fernández Lorences (2011: 194, base de comparación). 
Una base contrastiva compónenla dos términos contrastivos (TC), que son les formes llingüístiques concretes que marquen la diferencialidá. Cuando un falante asturianu produz un discursu faláu híbridu, inevitablemente fai escoyetes llingüístiques pal llau asturianu o pal llau castellanu al atopase coles diverses bases contrastives.

Llamamos corpus contrastivu (CC) al conxuntu de les bases contrastives d'un testu; el CC ye la base pa los cálculos del índiz de proporción llingüística, que se verá más abaxo.

\section{Correctu determín de les bases contrastives d'un testu}

Un asuntu fundamental ye afitar correctamente les bases contrastives nun testu híbridu, partiendo de los dos lectos confrontaos. Si se trata d'atopar la proporción de trazos asturianos d'un testu, nun podremos operar de la mesma manera si'l lectu de base ye l'asturianu central nuclear o l'asturianu occidental.

Acordies colo dicho más atrás, nel presente ensayu nun intervién n'absoluto nengún factor perceptivu del falante. L'análisis, entós, ye bien diferente del propuestu n'Andrés (2002: 34), de tipu perceptivu; ellí suponíase que formes del amestáu como lloques o loques percíbense como asturianes, porque prevalez la marca morfolóxica -es y non la forma del lexema, que resultaría indiferente. Sin embargu, nel presente ensayu - nel que nun se fai ponderación nenguna según el tipu de trazos - la forma lloques contendría dos trazos asturianos (lexema / hok-/ y marca morfolóxica -es), mentanto que loques contendría un trazu castellanu (lexema /lok-/) y un trazu asturianu (marca morfolóxica -es).

\section{Bases bivalentes}

Nuna serie de casos, atopámonos con bases de dos opciones, una contrastiva (BC) y otra común (BCom). Llamámosles bases bivalentes (BB). Por exemplu, al respective del fenómenu «forma lexemática del sustantivu 'verdá'» puen considerase dos opciones: 
BC: ast. verdá II cast. verdad

BCom: ast. verdá = cast. verdá

Nel exemplu, la BC esplícase porque la forma cast. verdad ye susceptible d'apaecer como opción real, averada al rexistru estándar y realizada como [-ð] o como [- $\theta]$. El segundu supuestu, la BCom, esplícase porque nel castellanu coloquial norteñu, el qu'entra en contactu col asturianu, ye mui normal la forma ensin /-d/ final, en coincidencia col asturianu (Hernández Alonso, 1999: 200; Viejo Fernández, 2005: 142).

Otru exemplu en sen contrariu, referíu al fenómenu «forma del alverbiu 'equí'»:

$$
\begin{aligned}
& \text { BC: ast. equí Il cast. aquí } \\
& \text { BCom: ast. aquí = cast. } \text { aquí }
\end{aligned}
$$

Esta BB débese a que l'asturianu conoz dos formes autóctones pa esti alverbiu: equí y aquí.

\section{Corpus contrastivu sintéticu (de fenómenos) y corpus con- trastivu analíticu (d'ocurrencies)}

De la que s'establez el corpus contrastivu (CC) d'un testu, esti tien dos posibilidaes: CC sintéticu, que se refier namái a los fenómenos y prescinde del númberu d'ocurrencies que'l términu contrastivu escoyíu se repite a lo llargo del testu; o CC analíticu, qu'atiende a los términos contrastivos escoyíos coles sos ocurrencies y les sos repeticiones.

\section{Bases contrastives con términos contrastivos diverxentes}

Nun testu determináu hai bases contrastives qu'apaecen representaes por un mesmu términu contrastivu, pero otres apaecen representaes por TC diverxentes. Por exemplu, nun testu'1 
fenómenu «forma del lexema alverbial locativu 'onde'» pue tar representáu por 5 ocurrencies, 4 d'elles cola forma ast. ónde y 1 cola forma cast. dónde. L'índiz d'asturianidá del fenómenu (recuentu sintéticu) ye una fracción de la unidá, que s'obtién aplicando una razón de proporcionalidá sobre un denominador equivalente a 1: nel exemplu será un índiz d'asturianidá de $4: 5$ $=0,8$. Lóxicamente, del recuentu analíticu saldría simplemente un índiz d'asturianidá de 4, que ye'l númberu d'ocurrencies de la forma ónde.

\section{Trazos individuales}

Como se dixo atrás, nesti ensayu la unidá de cómputu ye o bien la base contrastiva o fenómenu diferencial (corpus contrastivu sintéticu), o bien les ocurrencies concretes de los términos contrastivos (corpus contrastivu analíticu). Yá seyan fenómenos o ocurrencies de casos concretos, tómense de manera individual ún a ún, ensin ponderar tipoloxíes (fonético-fonolóxicos, morfosintácticos o léxico-semánticos) nin combinaciones de tipoloxíes (por exemplu, determinaos lexemes con determinaes marques morfolóxiques, etc. $)^{11}$.

Esti ensayu tampoco nun propón un estudiu de les interferencies, nin de les regles que conformen el lectu híbridu, nin de les alternancies de códigos, si les hai: ye, simplemente, un recuentu d'unidaes que formen masa estadístico. D'esta manera, nuna secuencia como la mi fía nun nos interesen les peculiares pautes de combinación de trazos que lleven a esta forma híbrida (y que bloquien, por exemplu, la posibilidá de *mi fía), sinón el simple fechu de qu'esa forma contién cuatro bases contrastives realizaes por aciu de cuatro términos contrastivos: trés asturianos (artículu, tonicidá, lexema fí-) y ún castellanu (forma del posesivu mi).

11 Esto segundo ye lo que fai Prieto (1991: 22-23), qu'establez una tipoloxía combinatoria de formes híbrides formaes pola combinación de tipos de pleremes + tipos de formantes morfolóxicos, d'au salen 9 clases. 


\section{Contraste testual}

Nesti ensayu les bases contrastives establécense polos contrastes efectivos en testu. Por exemplu: una secuencia del tipu pan duro presenta, d'acuerdu col nuestru métodu, una base común, desque nun hai visible nengún contraste nin escoyeta diferencial de llingua; ye bien posible que'l falante, al emitir pan duro, escoyera -o como marca morfolóxica asturiana de neutru de continuidá, pero a últimes ye coincidente cola marca morfolóxica castellana -o de masculín. Sin embargu, una base contrastiva clara sería la qu'atopamos na secuencia ropa blanco, onde tamos ante la BC «ast. ropa blanco I| cast. ropa blanca».

\section{Cuatro opciones de corpus contrastivos}

El propósitu d'esti ensayu ye atopar l'índiz de proporción testual del asturianu (índiz d'asturianidá testual) de dos testos de prueba, ún de Cabranes y otru de Xixón. Aplicando les opciones de recuentu descrites enriba, resulten cuatro opciones de corpus contrastivos, y polo tanto cuatro opciones de cálculu del mentáu índiz. Son los siguientes, presentaos de menor a mayor amplitú.

(1) Corpus sintéticu d'algame estrechu (CSAE): (a) considérense los fenómenos diferenciales con independencia del númberu d'ocurrencies de términos contrastivos; (b) los fenómenos con términos contrastivos (TC) diverxentes contabilícense col númberu fraccionariu que correspuenda al asturianu; (c) los fenómenos bivalentes descártense al interpretalos como bases comunes. Exemplos:

(a) El fenómenu «forma del pronome átonu de complementu indirectu na $3{ }^{\text {er }}$ persona del singular» tien un valor d'asturianidá de 1 anque apaeza cinco vegaes la forma ast. $-y$.

(b) El fenómenu «forma del alverbiu locativu 'ónde'», representáu con 4 TC ast. ónde y 1 TC cast. dónde, tien un valor d'asturianidá de 0,8 .

(c) El fenómenu de base bivalente «ast. verdá II cast. verdad verdá», representáu en testu por verdá, descártase del corpus, por considerase nesti casu una base común ast. verdá = cast. verdá. El fe- 
nómenu de base bivalente «ast. equí aquí I| cast. aquí», representáu en testu pola forma aquí, descártase del corpus por considerase la base común «ast. aquí = cast. aquí».

D'acuerdu colos exemplos, nel siguiente esquema márquense en cursiva versal les formes constataes de fechu nun testu, y en cursiva redondo les formes virtuales de contraste:

\begin{tabular}{c|c|c} 
asturianu & castellanu & $\begin{array}{c}\text { índiz } \\
\text { d'asturianidá }\end{array}$ \\
\hline$-Y$ & & \\
$-Y$ & & 1 \\
$-Y$ & $l e$ & \\
$-Y$ & & 0,8 \\
$-Y$ & & \\
\hline ÓNDE & & [fenómenu \\
ÓNDE & DÓNDE & \\
ÓNDE & & descartáu] \\
\hline ÓNDE & [fenómenu \\
descartáu]
\end{tabular}

(2) Corpus sintéticu d'algame ampliu (CSAA): (a) y (b), como nel casu anterior; (c) los fenómenos de base bivalente contabilícense cuando'1 TC escoyíu ye asturianu. Exemplos:

(a) y (b) Como nel casu anterior.

(c) Al fenómenu de base bivalente «ast. verdá II cast. verdad verdá» dáse-y el valor d'asturianidá 1 si ta representáu en testu pola forma verdá, por considerase agora la base contrastiva ast. verdá II cast. verdad. El fenómenu de base bivalente «ast. equí aquí II cast. aquí», representáu en testu pola forma aquí, descártase del corpus por considerase la base común «ast. aquí = cast. aquí». 


\begin{tabular}{c|c|c} 
asturianu & castellanu & $\begin{array}{c}\text { índiz } \\
\text { d'asturianidá }\end{array}$ \\
\hline$-Y$ & & \\
$-Y$ & & \\
$-Y$ & $l e$ & 1 \\
$-Y$ & & \\
$-Y$ & & \\
\hline ÓNDE & & \\
ÓNDE & DÓNDE & \\
ÓNDE & & \\
ÓNDE & & [fenómenu \\
\hline VERDÁ & verdad & descartáu
\end{tabular}

(3) Corpus analíticu d'algame estrechu (CAAE): (a) considérense los términos contrastivos escoyíos coles repeticiones que muestren en testu; (b) los términos de fenómenos bivalentes descártense si son interpretables como bases comunes. Exemplos:

(a) El fenómenu «forma del pronome átonu de complementu indirectu de 3 er $^{\text {er }}$ persona del singular» tien un valor d'asturianidá de 5 porque la forma ast. $-y$ apaez cinco vegaes en testu.

(b) El términu verdá descártase del corpus, por considerase nesti casu una base común ast. verdá = cast. verdá. El términu aquí descártase del corpus por considerase la base común «ast. aquí = cast. aquì».

\begin{tabular}{c|c|c} 
asturianu & castellanu & $\begin{array}{c}\text { índiz } \\
\text { d'asturianidá }\end{array}$ \\
\hline$-Y$ & & \\
$-Y$ & $l e$ & 5 \\
$-Y$ & & \\
$-Y$ & & \\
$-Y$ & & \\
\hline$O N D E$ & & \\
ÓNDE & DÓNDE & [ítem \\
ÓNDE & & descartáu] \\
\hline ÓNDE & [ítem \\
\multicolumn{2}{c}{ VERDÁ } & descartáu]
\end{tabular}


(4) Corpus analíticu d'algame ampliu (CAAA): (a) como nel casu anterior; (b) los términos de fenómenos bivalentes contabilícense cuando son interpretables con base contrastiva. Exemplos:

(b) L'ítem verdá cúntase con un valor d'asturianidá 1 por considerase la base contrastiva ast. verdá II cast. verdad. L'ítem aquí cúntase con un valor d'asturianidá 0 por considerase la base contrastiva «ast. equí I| cast. aquí».

\begin{tabular}{c|c|c} 
asturianu & castellanu & $\begin{array}{c}\text { índiz } \\
\text { d'asturianidá }\end{array}$ \\
\hline$-Y$ & & \\
$-Y$ & le & 5 \\
$-Y$ & & \\
$-Y$ & & \\
$-Y$ & & \\
\hline ÓNDE & & \\
ÓNDE & DÓNDE & \\
ÓNDE & & 1 \\
\hline ÓNDE & & 0
\end{tabular}

\section{Índiz de proporción testual. Índiz d'asturianidá testual}

L'indiz de proporción testual (IPT) d'un lectu o llingua $L$ ye la proporción de términos contrastivos qu'algama, nun testu híbridu, un lectu o llingua sobre'l conxuntu del corpus contrastivu. La fórmula ye esta:

$$
\mathrm{IPT}_{\mathrm{L}}=\frac{\mathrm{TC}_{\mathrm{L}} \cdot 10}{\mathrm{CC}}
$$

Que se llee asina: l'índiz de proporción testual d'un lectu o llingua L equival al númberu de términos contrastivos adscritos 
a L, multiplicáu pola base 10 y dividíu ente'1 númberu del corpus contrastivu del testu. Aplicao al asturianu (índiz d'asturianidá testual, IAT), nun hai más qu'interpretar l como asturianu.

Dao que, como se vio, consideramos cuatro opciones de corpus contrastivos, nel análisis d'un mesmu testu podrán obtenese cuatro IAT distintos. A últimes, faciendo la media de los cuatro IAT obtiénse un índiz mediu d'asturianidá testual, a mou de corolariu.

\section{Aplicación a dos testos concretos: Cabranes frente a Xixón}

$\mathrm{Al}$ envís de probar el métodu, escoyemos dos testos orales que presenten, a güeyu, diferencies notories. Ún, sacáu de la monografía de María Josefa Canellada El bable de Cabranes (Canellada, 1944: 53-54), ye la trescripción oral d'un cuentu cuntáu por una vecina de Madiéu que daquella tenía 84 d’años.

L'otru procede de los testos orales númb. 19 y númb. 21 recoyíos en Concha Prieto Investigación sociollingüística na Plazal Sur de Xixón (Prieto, 1991: 49-50 y 53-54, respectivamente), onde apaez la trescripción del falamientu espontaneu d'una vendedora de la Plaza'l Sur de Xixón, d'unos 65 años daquella.

Nes fontes orixinales, dambos testos apaecen en trescripción fonética, pero equí reproducímoslos n'ortografía normal.

\section{Testu de Cabranes}

Una vez morrió una muyer en Piedrafita ${ }^{12}$. El viudu alcontraba toeles coses feches cuando golviá pa casa pela tardiquina. «Descurro que... Ye la mio vecina la que me lo fai».

Y diba p'allá y dicía-y:

-Á mantina, Dios te lo pague, que alcuentro la torta cocín y el pote puestu.

-Nun, manti, yo non fúi. 
- ¡Ma! Entós ¿quién degorriu será?

Conque con eso un día igual y otru día igual. Una tardi llegól viudu más aína y alcontró en casa una mociquina mui guapa, piquiñina, que desamó pel mont'arriba acabantes de velu. Pero elli galguió tres d'ella a carrenderes y algamóla, y tréxola pa casa nun brazáu. Con eso, ella quedóse a vivir con elli, en faciendo esti tratu: «Más qu'oigues lo qu'oigues pel monte nun me digues nada». Conque al atardecerín del otru día preguntó-y ella:

-Á Xuaco, ¿qu'oísti pel monte, ho?

Yelli díxo-y:

- Oyí una voz que dicía: «Xana mana, ven enterrar a to ma(d)re ${ }^{13}$ qu'está finada» ${ }^{14}$.

Entós la xana apurrió-y con una restiella nes pates y el hombre daba unos apellidíos "Ai, que me matasti». Y la xana, desamando pel mont'arriba, dicía-y: «Pon llanzuela y quita llanzuela, y verás cómo suelda». Y sumióse pel mont'arriba y desapaició l'encantu.

\section{Rexistru de bases y términos contrastivos del testu de Cabranes}

Tanto nesti casu como nel siguiente de Xixón, les bases contrastives presentámosles nuna sucesión fonética-fonoloxía - morfosintaxis y léxicu nominal - y morfosintaxis y léxicu verbal; indíquense siempre les bases bivalentes (BB). El términu contrastivu realizáu efectivamente en testu va destacáu en versal, destacando con sorrayáu'l trazu concretu; el términu contrastivu virtual vien conseñáu en cursiva sin versal. Dacuando inclúinse esplicaciones llingüístiques sobre la tipificación.

1. Inestabilidá de vocales átones anteriores [BB].

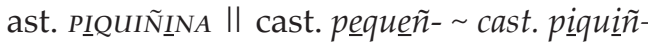

$$
\begin{aligned}
& \text { ast. DEsSCURRo } \| \text { cast. discurro } \sim \text { descurro }
\end{aligned}
$$

$13 \mathrm{Nel}$ orixinal [máre]. Forma inusitada nel propiu Cabranes (y en xeneral nel asturianu), que nun recueye'l vocabulariu de la monografía, que namái rexistra ma. El fechu d'apaecer nuna fórmula d'esconxuru pue esplicar esta singularidá.

14 Nel orixinal [fináıa]. 
El castellanu, en rexistros coloquiales o populares, nun ye ayenu a esta inestabilidá de vocales átones (Hernández Alonso, 1999: 198-199).

2. Vocal átona final.

$$
\text { ast. TARDI I| cast. tarde }
$$

N'asturianu centro-oriental ye mui corriente la variante tardi, non recoyida nel estándar (tarde).

3. Comportamientu de /-d-/ intervocálicu na secuencia -ad- de sufixu sustantivu [BB].

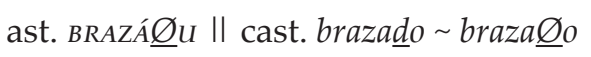

La cayida de /-d-/ na terminación -ado ye corriente nel castellanu coloquial norteño (Hernández Alonso, 1999: 199; Viejo Fernández, 2005: 142).

4. Comportamientu de /-d-/ intervocálicu na secuencia -id- de sufixu sustantivu d'orixe participial.

ast. cocí $\underline{u} u$ II cast. -id

ast. APELLIDÍ $\underline{\text { Oos }}$ I| cast. -id

Nesta terminación, el castellanu norteñu nun pierde'l fonema /-d-/.

5. Prosodia acentual del posesivu prenuclear.

ast. LA MIO VECINA I| cast. $\underline{\text { mi vecina }}$

ast. TO MA(D)RE I| cast. tu madre

El contraste vien determináu pola tonicidá del posesivu n'asturianu y la so atonicidá en castellanu.

6. Prosodia acentual d'una forma verbal del verbu 'ser'.

ast. F $\underline{\underline{u} I} I \|$ cast. $f u \underline{i}$

7. Alomorfu del artículu masculín singular ante vocal.

ast. l'home II cast. $\underline{E L}$ HOMBRE

ast. $\underline{L}^{\prime} E N C A N T U \|$ cast. $\underline{\text { el encanto }}$

8. Marca morfolóxica de masculín singular nos sustantivos.

ast. viUD $\underline{u} \|$ cast. viudo

ast. VIUD $\underline{u} \|$ cast. viud $\underline{0}$

ast. BRAZÁu $\|$ cast. brazado 
ast. TRAT $\underline{\underline{U}}$ II cast. trato

ast. ENCANT $\underline{u}$ \| cast. encanto

No que se refier al asturianu, paez conveniente estremar la $-u$ de sustantivos de la d'axetivos o pronomes, desque la casuística non siempre coincide: hai una serie de sustantivos asturianos que tienen una - $O$ autóctona en masculín singular (anque nesti testu nun apaez nengún) ${ }^{15}$.

9. Marca morfolóxica de masculín singular nos axetivos.

ast. EL POTE PUEST $\underline{U}$ || cast. puesto

ast. OTR $\underline{u}$ DÍA || cast. otro

ast. DEL OTR $\underline{\underline{U}}$ DÍA $\|$ cast. otr $\underline{\underline{O}}$

La secuencia la tarta cocíu y el pote puestu convida a analizar puestu como xéneru neutru o de continuidá, acordies col sistema centrooriental y oriental masc. $-u$ / fem. $-a$ / neutru $-u$, propiu de Cabranes. Sin embargu, al coincidir equí - $u$ del neutru col masculín (vasu puest $\underline{u}$, por exemplu), namás consideramos un claru contraste de neutru en secuencies del tipu torta cocíu.

10. Marca morfolóxica de masculín singular nos pronomes de complementu directu.

$$
\text { ast. VEL } \underline{\underline{u}} \text { | | cast. verlo }
$$

11. Marca morfolóxica de femenín plural correspondiente a singular en $-a$.
ast. TOE(S) I| cast. tod $\underline{\underline{S}}$
ast. L $\underline{E S}$ \| cast. las
ast. $\cos \underline{\underline{E S}} \|$ cast. cos $\underline{\mathrm{aS}}$
ast. FECHES $\|$ cast. hech $\underline{a S}$
ast. A CARRENDERES I| cast. a carreras

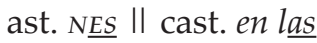
ast. PATES $\|$ cast. patas

Nel testu apaez la secuencia toeles, qu'a efectos d'esti análisis refáise como toe(s); l'asimilación de /-s/ a /-1/ nesi contestu nun ye di-

15 Ver García Arias (2003: 19-22 y 133-155). 
ferencial, porque dase tamién nel castellano coloquial (toalas). Ast. $a$ carrenderes fórmase dende'1 sustantivu carrendera 'carrera'.

12. Marca de neutru o xéneru de continuidá nos axetivos. ast. LA TORTA COCí́ || cast. la torta cocida

En Cabranes el sistema de xéneru nel axetivu ye de tipu oriental ${ }^{16}$ : masc. $-u$ / fem. $-a /$ neutru $-u$, frente al central $-u /-a /-o$.

13. Marca de masculín singular nel demostrativu. ast. ESTI I| cast. este $\underline{e}$

14. Forma del posesivu de 1. a persona del singular. ast. LA MIO VECINA I| cast. $\underline{\text { mi vecina }}$

15. Forma del posesivu de 2. ${ }^{\text {a }}$ persona del singular. ast. $\underline{T O} M A(D) R E ~ I \mid ~ c a s t . \underline{t u}$ madre

16. Artículu col posesivu prenuclear. ast. LA MIO VECINA I| cast. $\underline{\varnothing}$ mi vecina

17. Forma del lexema nominal el indefiníu 'tou'. ast. $\underline{\operatorname{TOE}(\mathcal{S})}$ II cast. $\underline{\text { todas }}$

18. Forma del masculín singular del pronome tónicu de suxetu de 3. ${ }^{a}$ persona del singular.

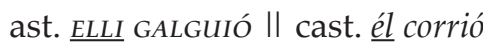

ast. CON $\underline{E L L I} \|$ cast. con $\underline{e}$

ast. Y $\underline{E L L I} \|$ cast. $y \underline{e ́ l}$

19. Forma del pronome átonu de complementu indirectu en singular.

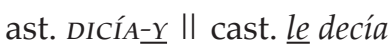

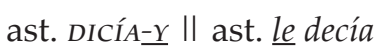

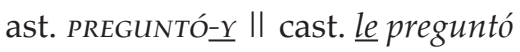

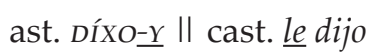

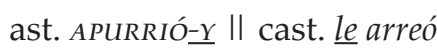


20. Enclisis de los pronomes átonos con formes conxugaes d'indicativu.

ast. DICÍA $\underline{\underline{Y}}$ \| cast. le decía

ast. ALGAMÓLA $\|$ cast. la alcanzó

ast. TRÉXOLA $\|$ cast. $\underline{\text { la }}$ trajo

ast. QUEDÓsE II cast. $\underline{\text { se quedó }}$

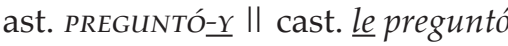

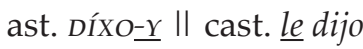

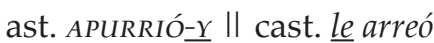

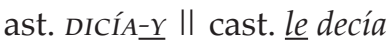

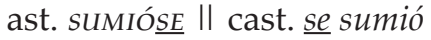

Equí tenemos en realidá un contraste ente dos sistemes d'allugamientu de los pronomes clíticos dafechamente diferentes. Agora, na realización en testu, el contraste produzse namás naquellos casos onde l'asturianu amuestra enclisis con formes verbales conxugaes. Y asina, los casos de proclisis asturiana como la que me lo fai, Dios te lo pague, que me matasti, anque obedecen a razones sintáctiques mui diferentes a los correspondientes exemplos en castellanu, preséntense como Base Común a efectos d'análisis contrastivu testual ${ }^{17}$.

21. Forma de la negación.

ast. $\underline{N U N}$, MANTI $\|$ cast. $\underline{\text { no }}$, cariño

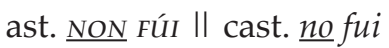

ast. NUN ME DIGUES I| cast. $\underline{\text { no }}$ me digas

Nos testos recoyíos en Cabranes por Canellada nun hai un repartu de negaciones como n'otres zones del asturianu y nel estándar, del tipu nun + verbu frente a non n'otros contestos. Equí hai una indistinción que paez llevar a nun o non albidramos que por razones de comportamientu prosódicu de la vocal. En tou casu, los trés exemplos d'esti testu caltienen el trazu diferencial col castellanu.

17 Pal allugamientu de los pronomes clíticos col verbu, ver Andrés (1993) y GLA (2001: 366-357). 
22. Forma del alverbiu 'entós'.

ast. ENTÓs II cast. entonces

ast. ENTós II cast. entonces

23. Unidá léxica alverbial.

ast. AÍNA I| cast. pronto

24. Asimilación preposicional de 'per', 'por' col artículu [BB].

ast. PELA TARDIQUINA I| cast. por la $\sim$ pola

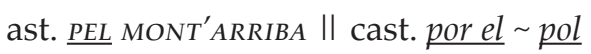

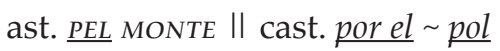

ast. PEL MONTE II cast. por el $\sim$ pol

ast. PEL MONT'ARRIBA \|l cast. por el $\sim$ pol

ast. PEL MONT'ARRIBA II cast. por $\mathrm{el} \sim$ pol

El castellanu coloquial norteñu tamién conoz esa mena d'asimilaciones.

25. Asimilación preposicional de 'en' col artículu.

ast. NES PATES II cast. en las

El castellanu coloquial norteñu nun conoz estes asimilaciones.

26. Alomofu de la preposición 'en' ante vocal.

ast. NUN BRAZÁU II cast. en un brazado

27. Forma de la preposición 'pa' [BB].

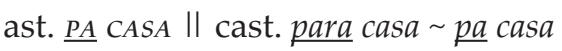

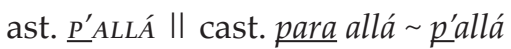

ast. PA CASA II cast. para casa pa casa

La doble posibilidá (ast. pa || cast. para; ast. $p a=$ cast. $p a$ ) vien del fechu de que nel castellanu coloquial norteñu ye mui usada tamién la forma $p a$.

28. Forma de la preposición 'tres'.

ast. $\underline{\text { TRES }}$ || cast. tras 
29. Combinación preposicional 'tres $+\mathrm{de}^{\prime}$.

ast. $\underline{\text { TRES D' }}$ ELLA \| cast. tras ella

30. Unidá preposicional.

ast. PELA TARDIQUINA I| cast. por la tardecita

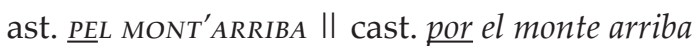

ast. PEL MONTE I| cast. por el monte

ast. PEL MONTE I| cast. por el monte

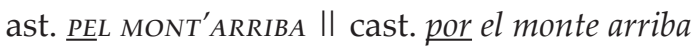

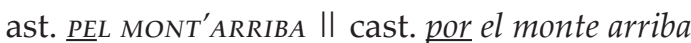

Frente a la preposición cast. por, l'asturianu centro-oriental tien dos unidaes preposicionales con distintes formes y usos: por y per, esta con valor locativu, temporal y instrumental. En testu, por razones de fonética sintáctica vistes enriba, nun se presenta na forma plena.

31. Forma de conxunción adversativa [BB].

ast. MÁS QU'OIGUES $\|$ cast. aunque oigas $\sim$ más que oigas

Si consideramos como conxunción adversativa normal cast. aunque, produzse un contraste cola forma asturiana; pero'l castellanu tamién conoz la forma más que, polo que se produz tamién una Base Común. La grafía normativa n'asturianu ye masque.

32. Construcción conxuntiva temporal.

ast. ACABANTES DE VELU \| cast. nada más verlo

33. Unidá interxectiva vocativa.

ast. $\underline{\mathrm{HO}}$ \|l cast. tú; oye...

34. Unidá interxectiva vocativa.

ast. $\underline{A}$ MANTINA I| cast. oye, cariñito

ast. $\underline{A}$ XuAco II cast. oye, Joaquín

35. Unidá interxectiva espresiva.

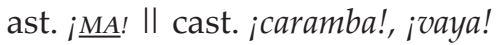


36. Marca morfolóxica de 2. ${ }^{a}$ persona del singular del presente de suxuntivu de verbos de la 2. ${ }^{\mathrm{a}}$ y $3 .{ }^{\mathrm{a}}$ - conxugaciones.

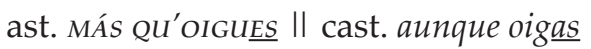

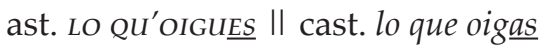

$$
\begin{aligned}
& \text { ast. NUN ME DIGUE } \text { | | cast. no me digas }
\end{aligned}
$$

37. Marca morfolóxica de 2. ${ }^{a}$ persona del singular del pretéritu indefiníu.

$$
\begin{aligned}
& \text { ast. oÍst II || cast. oíste } \\
& \text { ast. MATASTI I| cast. mataste }
\end{aligned}
$$

38. Tiempu simple y tiempu compuestu con 'haber': pretéritu indefiníu simple y pretéritu perfectu compuestu [BB].

$$
\begin{aligned}
& \text { ast. } \underline{\underline{\text { Eú }}} \| \text { cast. he sido } \sim \text { fui } \\
& \text { ast. oísTI II cast. has oído } \sim \underline{\text { oíste }} \\
& \text { ast. oYí } \| \text { cast. he oído } \sim \underline{\text { oí }} \\
& \text { ast. MATASTI II cast. has matado } \sim \text { mataste }
\end{aligned}
$$

Magar que nestos casos paez asumible qu'en castellanu l'usu previsible sería'l pretéritu perfectu compuestu, nun se pue refugar un usu tamién normal del pretéritu indefiníu simple nos mesmos contestos. En resultes, contemplamos la doble opción d’análisis.

39. Forma de la $3 .^{a}$ persona del singular del presente d'indicativu del verbu 'ser'.

$$
\text { ast. } \underline{Y E} \| \text { cast. } \underline{e S}
$$

40. Alomorfu del lexema del verbu 'facer' en delles formes del presente d'indicativu.

$$
\text { ast. } \underline{\underline{F A I}} \text { II cast. } \underline{\text { hace }}
$$

41. Alomorfu del lexema del verbu 'facer' en xerundiu. ast. FACIENDO II cast. haciendo

42. Alomorfu del lexema del verbu 'facer' en participiu. ast. $\underline{\text { FECHES }}$ I| cast. hechas 
43. Alomorfu del lexema del tema de presente del verbu 'dicir' [BB].

ast. DICÍA-Y \|| cast. le decía le dicía

ast. DICíA-Y II cast. le decía le dicía

ast. QUE DICía II cat. que decía que dicía

La bivalencia nesti casu produzse porque'l castellanu norteñu conoz tamién, polo menos en dellos rexistros, un lexema con forma dic(Hernández Alonso, 1999: 198-199).

44. Forma del lexema del tema de perfectu del verbu 'dicir'.

ast. $\underline{\text { DÍxO}} \mathrm{x}$ Y II cast. le dijo

45. Forma del lexema del tema de perfectu del verbu 'trayer'.

ast. TRÉXOLA II cast. la trajo

46. Comportamientu de la /-r/ final del infinitivu con un pronome enclíticu.

ast. $V E \underline{\complement} \underline{L U} \|$ cast. verlo

47. Construcción temporal 'en' + xerundiu [BB].

ast. EN FACIENDO I| cast. nada más hacer $\sim \underline{\text { en haciendo }}$

El castellanu, polo menos en dellos rexistros, conoz tamién tala construcción, polo que contemplamos tamién una Base Común.

48. Preposición 'a' en complexu verbal 'venir' + infinitivu.

ast. VEN $\underline{\varnothing}$ ENTERRAR I| cast. ven a enterrar

49. Forma del lexema del sustantivu 'muyer'.

ast. MUYER I| cast. $\underline{\text { mujer }}$

50. Forma del lexema del sustantivu 'home'.

ast. home II cast. $\underline{\text { HOMBRE }}$

51. Forma del lexema del nome propiu ' $X u a c u$ '

ast. XuAco II cast. Joaquín, Juaco

52. Significáu del sustantivu 'pata' [BB].

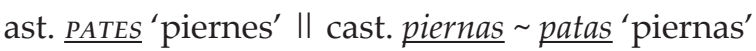


El significáu 'piernas d'una persona' tamién ye conocíu en rexistros coloquiales del castellanu (DRAE, sv).

53. Unidá léxica de sustantivu.

ast. MANTINA I| cast. cariñito

ast. $\underline{\text { MANTI }} \|$ cast. cariño

54. Unidá léxica de sustantivu.

ast. $\underline{\text { pOTE }} \|$ cast. cocido, olla

L'acepción de pote que recueye'1 DRAE 'comida equivalente en Galicia y Asturias a la olla de Castilla' demuestra la so condición de palabra asturiana.

55. Unidá léxica de sustantivu [BB].

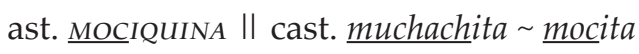

Ye posible establecer una BC que contrapón ast. moza a cast. muchacha; en tou casu, moza tamién ye castellanu, y anque d'un usu más marcáu en relación con dellos rexistros, ye posible considerar tamién una Base Común.

56. Unidá léxica d'axetivu [BB].

ast. GUAPA II cast. bonita guapa

Dos opciones: una BC dafechamente verificable ast. guapu $-a-0$ II cast. bonito $-a$; o una BCom ast. guapu $-a-o=$ cat. guapo $-a$, desque esti axetivu pue, en dellos rexistros, presentar el mesmu significáu que n'asturianu (DRAE).

57. Unidá léxica de sustantivu.

ast. $\underline{\underline{X N A} A} \|$ cast. $\varnothing$

ast. LA $\underline{\text { XANA }}$ || cast. $\varnothing$

ast. $Y_{\text {LA }} \underline{\mathrm{X} A N A} \|$ cast. $\varnothing$

58. Unidá léxica de sustantivu.

ast. $\underline{\text { MANA }}$ || cast. hermana

En realidá, ast. mana (d'hermana) tien equí un usu mui específicu nuna fórmula d'esconxuru. 
59. Unidá léxica de sustantivu.

ast. RESTIELLA I| cast. cardadora, tabla de cardar

60. Unidá léxica de sustantivu.

ast. APELLIDÍOS I| cast. gritos

61. Unidá léxica de sustantivu.

ast. PON LLANZUELA II cast. pon llantén

ast. QUITA LLANZUELA I| cast. quita llantén

62. Unidá léxica de sustantivu.

ast. DEGORRIU || cast. demonio

63. Unidá sufixal de diminutivu.

ast. TARDIQUINA $\|$ cast. tardecita

ast. MOCIQUINA I| cast. muchach $\underline{i t a}$

64. Unidá sufixal de diminutivu [BB].

ast. MANTINA || cast. $-\underline{i t o} \sim-\underline{i n}$

ast. PIQUIÑINA $\|$ cast. $-\underline{i t a} \sim-\underline{i n a}$

ast. AL ATARDECERIIN || cast. $-\underline{i t o} \sim-\underline{i n}$

Xuégase equí con dos posiblidaes: una BC ast. -ín -ina | | cast. -(ec) ito - $a$; o una BCom ast. -ín -ina = cast. -ín -ina, desque esti sufixu tien tamién dél usu nel castellanu norteñu.

65. Unidá fraseolóxica alverbial.

ast. A CARRENDERES $\|$ cast. a carreras

66. Forma del lexema del verbu 'alcontrar'.

ast. $\underline{\text { ALCONTRABA }}$ || cast. encontraba

ast. $\underline{\text { ALCUENTRO II cast. encuentro }}$

ast. $\underline{\text { ALCONTRÓ }}$ || cast. encontró

67. Forma del lexema del verbu 'morrer'.

ast. MORRIÓ || cast. $\underline{\text { murió }}$

68. Forma del lexema del verbu 'volver' [BB].

ast. GOLVIÁ II cast. $\underline{\text { volvía } ~ g o l v i ́ a}$ 
Nun tomamos en cuenta la desinencia -iá en cuentes del normal -ía (golvía), porque'l diptongu qu'equí se forma ha debese a circunstancies elocutives. En cuantes al lexema golv-, bien corriente en rexistros rurales del asturianu (xunto con volv-), pue contrastase col cast. volv-, pero pue igualase tamién con cast. golv-, presente en dellos rexistros non normativos.

69. Forma del lexema del verbu 'dir'.

ast. $\underline{\text { DIBA }} \|$ cast. $\underline{i b a}$

70. Forma del lexema del verbu 'oyer'.

ast. $\underline{\underline{o x i}} \|$ cast. $\underline{\underline{o}}$

En testu apaez tamién la forma ast. oísti, que ye coincidente col castellanu no que se refier al lexema (non en cuantes a la desinencia).

71. Forma del lexema del verbu 'tar'.

ast. $\underline{t} a \|$ cast. $\underline{E S T A ́}$

72. Forma del lexema del verbu 'desapaecer'.

ast. DESAPAICIÓ II cast. desapareció

73. Unidá léxica de verbu.

ast. DESAMÓ II cast. Ø 'corrió, escapó'

ast. DESAMANDO $\|$ cast. $\varnothing$ 'corriendo, escapando'

74. Unidá léxica de verbu.

ast. GALGUIÓ II cast. Ø 'corrió mucho'

75. Unidá léxica de verbu.

ast. ALGAMÓLA II cast. la alcanzó

76. Unidá léxica de verbu.

ast. APURRIÓ-Y I| cast. le arreó

77. Significáu verbal.

ast. SUMı́óse I| casta. desapareció 


\section{Índiz d'asturianidá del testu de Cabranes}

El testu de Cabranes representa una mena de fala claramente asturiana poles sos característiques llingüístiques; podría clasificase fácilmente como asturianu total, acordies colos criterios espuestos n'Andrés (2002). N'efectu, presenta trazos-frontera propios del asturianu mínimu: marca $-u$ de masculín singular, marca -es de femenín plural, diminutivu en -ín -ina, posesivu prenuclear con artículu, pretéritu indefiníu simple, forma verbal ye, perda de $-r$ de infinitivu con pronome enclíticu y sistema asturianu d'allugamientu de pronomes clíticos. Pero, amás, tien toa una riestra de trazos suplementarios asturianos, propios del asturianu total, ente otros: xéneru neutru de continuidá, verbu morrer, verbu alcontrar, verbu facer (fai, feches), verbu dir, negación non nun, preposición per, interxección á, vocativu manti, léxicu (galguió, algamó, restiella, desamó, apurrió, aína), etc.

Sometíu'l testu al procedimientu de cómputu propuestu, resulta:

(1) Corpus sintéticu d'algame estrechu (CSAE)

Bases contrastives del corpus: 65

Términos contrastivos asturianos: 62,5

Índiz d'asturianidá testual: 9,61

(2) Corpus sintéticu d'algame ampliu (CSAA)

Bases contrastives del corpus: 77

Términos contrastivos asturianos: 74,5

Índiz d'asturianidá testual: 9,67

(3) Corpus analíticu d'algame estrechu (CAAE)

Bases contrastives del corpus: 114

Términos contrastivos asturianos: 111

Índiz d'asturianidá testual: 9,73

(4) Corpus analíticu d'algame ampliu (CAAA)

Bases contrastives del corpus: 141 
Términos contrastivos asturianos: 138

Índiz d'asturianidá testual: 9,78

(5) Índiz mediu d'asturianidá testual: 9,69

\section{Testu de Xixón ${ }^{18}$}

Tu veslu por algún lau, no se puede creer.

No ye un vestidu, míralu ahí.

No lo sé.

Qué iría a meter la plancha aquí.

Y estaba un rapaz así, que parecía que me miraba, un rapaz relativamente joven, y dije yo: «Igual m'está mirando, porque me ve que hablo sola...». Diz él: «No, la verdá, estaba mirándola porque no sé por qué...». Dije yo: «Si supiera lo que me pasa...». Diz él: «¿Qué-y pasa, muyer?». Dije yo: "Llevo vente minutos buscando dónde aparquél coche y no soi a dar con él. Mire, sé que lu aparqué en tres sitios, y d'estos tres sitios lu tuve que sacar ${ }^{19}$ : de uno, porque me quedaba la culera mui afuera; del otru, porque no me cogía pa entrar del todo; y del terceru, después de que lu tenía bien aparcáu, un portón "por favor, no aparquen delante"»"20. Dije yo: «Y la cuarta vez que lu aparqué, ahora no sé dónde coño lu aparqué, y llevo vente minutos: la Residencia Robledo, por junto al Goya, por la calle Bermúdez...»

Pues nada, vine pa la plaza a decí-yoslo, porque iba a recoger una mercancía a Roces. Estaben esperando en la puerta l'autobús, y quedé d'estar allí d'once a once y media, y no me gusta decir aquí y llegar allá, pero tú querrás creer que lo anduve todo y no encuentro el coche... No sé dónde

18 Esti testu ta formáu polos falamientos d'una mesma persona dientro dos diálogos. Los falamientos de les otres persones elimínense equí por innecesarios.

19 ...d'estos tres sitios lu tuve que sacar. Magar que nel testu funciona'1 sistema asturianu d'allugamientu de clíticos, nesti exemplu $l u$ va proclíticu por focalización preverbal del sintagma d'estos tres sitios (Andrés, 1993: 37-43, 47-48; GLA, 2001: 366-357). Esti allugamientu proclíticu opera, a efectos de cómputu, como una base común col castellanu (de estos tres sitios lo he tenido que sacar).

20 Esta mención metallingüística a un testu en castellanu queda fuera del análisis. 
coño lu dejé... La mi fía: «Mamá, ¿no te lu llevaría la grúa?». Y digo yo: «No, donde yo lu dejo no me lu lleva la grúa». Pero no sé, no me vien a la imaginación. Mira, una vez salí por aquí... La segunda vez salí por aquí, no hice más que poneme en la esquina de Tesier y dije yo: "Ai, ya sé ónde lu dejé: ahí junto al juzgao, en esa plazoleta, ahí mismo». En la esquina del todo había un cachín de sitiu y tuve que metelu un poco encima la cera, pero dije: "Ya no ando más, aquí mismo». Pues no fue más que... Fíjate, ahí a dos pasos.

\section{Rexistru de bases y términos contrastivos del testu de Xixón}

1. Comportamientu de /-d/ final en sustantivos [BB].

ast. VERDÁØ I| cast. verda $\underline{\underline{d}} \sim \operatorname{verdá\varnothing }$

Pal castellanu pue considerase términu contrastivu la forma estándar verdad, con realización como [-ð] o [- $\theta]$, o bien el más corriente nes variedaes coloquiales verdá (Hernández Alonso, 1999: 200; Viejo Fernández, 2005: 142).

2. Comportamientu de /-d-/ intervocálicu na secuencia -ad- de terminación sustantiva [BB].

ast. $L A \varnothing u \|$ cast. la $\underline{d} o \sim$ la $\underline{\varnothing} 0$

ast. APARCÁØu II cast. aparcado aparcaØo

ast. JUzGAØo II cast. juzgado juzgaØo

Les variantes en -ado o en -ao son igualmente castellanes (Hernández Alonso, 1999: 199; Viejo Fernández, 2005: 142).

3. Comportamientu de /-d-/ intervocálicu na secuencia -id- de terminación sustantiva.

ast. vistíØ-, vestíØ- II cast. VESTID-

Nesti casu trátase d'una clara BC, porque'l castellanu coloquial norteñu nun pierde /-d-/ nesti contestu.

4. Prosodia acentual del posesivu prenuclear.

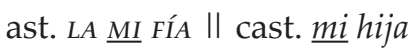

El posesivu prenuclear ye tónicu n'asturianu y átonu en castellanu.

5. Marca morfolóxica $-u$ de masculín singular nos sustantivos.

ast. LA $\underline{\underline{u}}$ II cast. lado la $\underline{\underline{O}}$ 
ast. VESTID $\underline{u}$ || cast. vestido

ast. SITI $\underline{\underline{u}} \|$ cast. sitio

ast. xuzgáu, xulgáu $\|$ cast. JUzGAQ

Al respective de $-u$ como marca de masculín singular, estremamos sustantivos d'axetivos, porque l'asturianu central almite una serie de sustantivos masculinos con marca $-o$, mentanto que los axetivos masculinos tienen mui fixada la terminación $-u$.

6. Marca morfolóxica $-u$ de masculín singular nos axetivos.

ast. DEL OTR $\underline{\underline{u}}$ \| cast. del otro

ast. DEL TERCER $\underline{\underline{u}}$ \| cast. del tercer $\underline{\underline{O}}$

ast. APARCÁu $\|$ cast. aparcado

7. Marca morfolóxica $-u$ de masculín singular nel pronome átonu de complementu directu.

ast. VESLL $\|$ cast. $\underline{\text { lo ves }}$

ast. MÍRALU $\|$ cast. míralo

ast. QUE LU APARQUÉ I| cast. que lo aparqué

ast. $\underline{L U}$ TUVE I| cast. $\underline{\text { lo tuve }}$

ast. DE QUE LU TENÍA II cast. de que lo tenía

ast. QUE LU APARQUÉ II cast. que lo aparqué

ast. DÓNDE COÑO LU APARQUÉ II cast. dónde coño lo aparqué

ast. DÓNDE COÑO LU DEJÉ I| cast. dónde coño lo dejé

ast. NO TE LU LLEVARÍA II cast. no te lo llevaría

ast. DONDE YO $\underline{L U}$ DEJO $\|$ cast. donde yo $\underline{\text { lo dejo }}$

ast. NO ME LU LLEVA \| cast. no me lo lleva

ast. ÓNDE LU DEJÉ || cast. dónde lo dejé

ast. METELLU $\|$ cast. meterlo

8. Marca morfolóxica de masculín singular nel indefiníu 'un'.

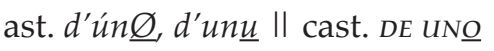


9. Forma del posesivu prenuclear de 1. ${ }^{\text {a }}$ persona del singular.

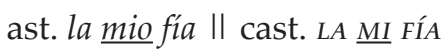

10. Artículu col posesivu prenuclear.

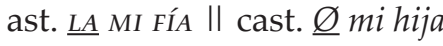

11. Forma del indefiníu 'dalgún' [BB]. ast. dalgún llau algún llau II cast. ALGÚN LAU.

Caben dos posibilidaes: la BC ast. dalgún frente a cast. algún; y la BCom ast. algún = cast. algún.

12. Forma del indefiníu 'mesmu'. ast. ehí mesmo || cast. AHÍ $\underline{\text { MISMO }}$ ast. equí mesmo II cast. AQUÍ MISMO

13. Forma del indefiníu 'tou'. ast. del to $\underline{\text { too }}$ || cast. DEL $\underline{\text { TODO }}$ ast. $\underline{\text { too }}$ II cast. $\underline{\text { TODO }}$ ast. del tooo || cast. DEL $\underline{\text { TODO }}$

14. Forma del interrogativu o relativu locativu. ast. ónde aparquél coche \| cast. DÓNDE APARQUÉ'L COCHE ast. ónde coño lu aparqué I| cast. DÓNDE COÑO LU APARQUÉ ast. ónde coño lu dejé I| cast. DÓNDE COÑO LU DEJÉ ast. onde yo lu dejo II cast. DONDE YO LU DEJO ast. ÓNDE LU DEJÉ II cast. dónde lu dejé

15. Forma de pronombe átonu de complementu indirectu en singular. ast. QUÉ-Yy PASA II cast. qué le pasa

16. Forma del pronome átonu de complementu indirectu en plural. ast. DECÍ-Y $\underline{\underline{Y}} L \mathrm{~S} L O \|$ cast. $\underline{\text { les }}$

17. Alomorfu del pronome átonu de complementu en singular y plural en combinación cola 3. ${ }^{\text {a }}$ persona del pronome átonu de complementu directu.

ast. DECÍ-YOSLO II cast. decírselo 
18. Enclisis de los pronomes átonos con formes conxugaes d'indicativu.

ast. VESLLU $\|$ cast. $\underline{\text { lo }}$ ves

19. Forma del alverbiu 'asina'.

ast. $\underline{\text { asina }}$ (ansí, ansina...) II cast. $\underline{A S i ́}$

20. Forma del alverbiu 'agora'.

ast. agora $\|$ cast. $\underline{A H O R A}$

21. Forma del alverbiu 'depués' [BB].

ast. depués (dempués) después II cast. DESPUÉS

Dos posibilidaes de cómputu, yá que después pue interpretase tamién como forma común.

22. Forma d'alverbiu locativu [BB].

ast. $\underline{e h i ́} \sim \underline{\text { ahí }}$ II cast. $\underline{A H i ́}$

ast. $\underline{\text { ehí }} \sim \underline{\text { ahí }}$ II cast. $\underline{A H I}$

ast. $\underline{\text { ehí }} \sim \underline{\text { ahí }} \|$ cast. $\underline{A H i ́}$

ast. $\underline{\text { ehí }} \sim \underline{\text { ahí }}$ II cast. $\underline{A H I}$

Danse dos opciones d'análisis: o bien hai una BC ast. ehí I I cast. ahí, y polo tanto ahí interprétase como términu castellanu; o bien hai una BCom ast. ahí $=$ cast. ahí.

23. Forma d'alverbiu locativu [BB].
ast. equí $\sim$ aquí || cast. $\underline{\text { AQuí }}$
ast. aquí $\sim \underline{\text { equí }} \|$ cast. $\underline{\text { AQuí }}$
ast. $\underline{\text { aquí }} \sim \underline{\text { equí }} \|$ cast. $\underline{\text { AQuí }}$
ast. $\underline{\text { aquí }} \sim \underline{\text { equí }} \|$ cast. $\underline{\text { AQuí }}$
ast. aquí $\sim \underline{\text { equí }} \|$ cast. $\underline{\text { AQuí }}$

Danse dos opciones d'análisis: o bien hai una BC ast. equí I I cast. aquí, y polo tanto aquí interprétase como términu castellanu; o bien hai una BCom ast. aquí $=$ cast. aquí.

24. Forma d'alverbiu locativu [BB].

ast. allí $\sim$ ellí II cast. $\underline{\text { ALLí }}$ 
Danse dos opciones d'análisis: o bien hai una BC ast. ellí II cast. allí, y polo tanto allí interprétase como términu castellanu; o bien hai una BCom ast. allí = cast. allí.

25. Forma de la espresión alverbial 'xunto a'.

ast. xunto a (xunta) II cast. LUNTO AL GOrA

ast. xunto a (xunta) II cast. LUNTO AL JUZGAO

26. Forma de la negación verbal .

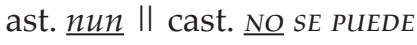

ast. $\underline{\text { nun }}$ || cast. $\underline{\text { NO }}$ ES

ast. $\underline{n U n}$ II cast. $\underline{\text { NO }}$ LO SÉ

ast. $\underline{\text { nUn }} \|$ cast. $\underline{\text { NO }} S E ́$

ast. $\underline{\text { nun }}$ || cast. $\underline{\mathrm{NO}}$ SOI

ast. $\underline{\text { nun }}$ || cast. $\underline{\text { NO ME COGÍA }}$

ast. $\underline{\text { nun }} \|$ cast. $\underline{\text { NO }}$ sÉ

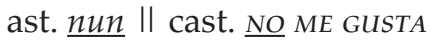

ast. $\underline{\text { nun }}$ \| cast. $\underline{\text { NO ENCUENTRO }}$

ast. $\underline{\text { nun }} \|$ cast. $\underline{\text { NO }} S E ́$

ast. $\underline{\text { nun }}$ II cast. ¿ ¿NO TE LU LLEVARÍA...?

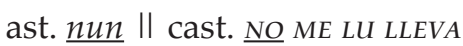

ast. $\underline{\text { nun }} \|$ cast. $\underline{\text { NO }} S E ́$

ast. $\underline{\text { nun }}$ I| cast. $\underline{\text { NO ME VIEN }}$

ast. $\underline{\text { nun }}$ || cast. $\underline{\text { NO HICE }}$

ast. $\underline{\text { nun }}$ || cast. $\underline{\text { NO ANDO }}$

ast. $\underline{\text { nun }}$ || cast. $\underline{\text { NO FUE }}$

27. Forma de la negación non verbal.

ast. $\underline{\text { non }}$ || cast. $\underline{\text { NO }}$ LA VERDÁ...

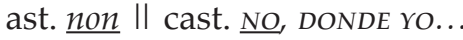

28. Forma de la preposición 'pa' [BB].

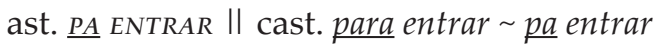


ast. PA LA PLAZA II cast. para la plaza pa la plaza

Hai dos posibilidaes d'análisis, darréu qu'en castellanu esta preposición tien dos formes alternantes: para nun rexistru estándar; y pa nos rexistros coloquiales.

29. Valor direccional de la preposición 'pa' [BB].

ast. PA LA PLAZA II cast. hacia la plaza para (pa) la plaza

Pue considerase un contraste ente ast. 'valor direccional espresáu con $p a^{\prime}$ frente a cast. 'valor direccional espresáu con hacia'; pero'l castellanu conoz tamién l'usu de para pa con esi valor, de manera que nesi casu interprétase una Base Común.

30. Asimilación preposicional de 'per', 'por' col artículu [BB].

ast. pela calle II cast. POR LA CALLE pola calle

31. Asimilación preposicional de 'en' col artículu.

ast. na puerta II cast. EN LA PUERTA

ast. na esquina || cast. EN LA ESQUINA

ast. na esquina II cast. EN LA ESQUINA

32. Alomorfu de la preposición 'de' ante vocal.

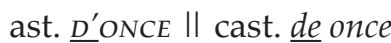

ast. $\underline{d^{\prime}} u ́ n, \underline{d^{\prime}} u n u$ II cast. $\underline{D E}$ UNO

Sicasí, na espresión d'estos nun interpretamos una BC, porque la xuntura vocálica de dos /e/ en contactu cúmplese normalmente nel castellanu faláu.

33. Alomorfu de preposición 'en' ante vocal.

ast. nesa plazoleta II cast. EN ESA PLAZOLETA [BB].

34. Supresión de la preposición 'de' n'axacente precedíu de vocal

ast. PUeRTA $\varrho$ L'Autobús II cast. puerta del autobús puerta $\varrho$ l'autobús

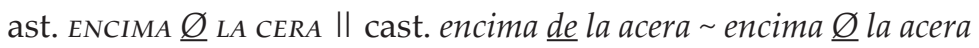

La supresión de de nesos contestos detéctase tamién nel castellanu coloquial (Hernández Alonso, 1999: 200). 
35. Unidá preposicional.

ast. per dalgún llau II cast. POR ALGÚN LAU

ast. per xunto al Goya II cast. POR JUNTO AL GOYA

ast. pela calle Bermúdez II cast. POR LA CALLE BERMÚDEZ

ast. per equí II cast. $\underline{\text { POR AQuí }}$

ast. per equí II cast. $\underline{P O R}$ AQUÍ

Frente a la preposición cast. por, l'asturianu centro-oriental tien dos unidaes preposicionales con distintes formes y usos: por y per, esta con valor locativu, temporal y instrumental. En testu, por razones de fonética sintáctica vistes enriba, preséntase en dellos casos con una forma asimilada al artículu.

36. Marca morfolóxica de 3. ${ }^{a}$ persona del plural del pretéritu imperfectu d'indicativu de los verbos de la 1. o conxugación.

ast. ESTABEN $\|$ cast. estaban

37. Marca morfolóxica de 1. a persona del singular del pretéritu indefiníu fuerte de dellos verbos irregulares.
ast. dixi $\|$ cast. DIJE YO
ast. dixi $\|$ cast. DIJE YO
ast. dixi $\|$ cast. DIJE Y YO
ast. tuvi $\|$ cast. LU TUVE
ast. dixi $\|$ cast. DIJE YO
ast. vini $\|$ cast. VINE
ast. anduvi II cast. QUE LO ANDUVE
ast. dixi $\|$ I cast. DIJE YO
ast. fici $\mid$ I | cast. HIC $\underline{E}$
ast. dixi $\|$ cast. PERO DIJE

38. Marca morfolóxica de 3. a persona del singular del presente d'indicativu del verbu 'venir'.

ast. VIEN $\underline{\varnothing}$ II cast. viene

39. Marca morfolóxica de 3. a persona del singular del presente d'indicativu del verbu 'dicir'. 
ast. DIZ $\underline{\emptyset} E ́ L$ || cast. dice

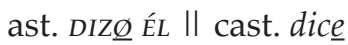

40. Forma del lexema del tema de perfectu del verbu 'dicir'.

ast. $\underline{\text { dixi }}$ II cast. DIIE YO

ast. $\underline{\operatorname{dix} i}$ II cast. DIIE YO

ast. $\underline{\text { dixi } i}$ II cast. DIIE YO

ast. $\underline{\text { dixi }}$ II cast. DIIE YO

ast. $\underline{\text { dixi }}$ II cast. DIIE YO

ast. $\underline{\text { dixi }}$ II cast. PERO $\underline{\text { DIJE }}$

41. Forma de la 3. ${ }^{a}$ persona del singular del presente d'indicativu del verbu 'ser'.

ast. $\underline{Y E} \|$ cast. $\underline{\underline{e}}$

42. Alomorfu del lexema del verbu 'querer' en futuru.

ast. quedrás II cast. QUERRÁs

43. Tiempu simple y tiempu compuestu con ‘haber': pretéritu indefiníu simple y pretéritu perfectu compuestu [BB] .

ast. DÓNDE APAROUÉ'L COCHE II cast. he aparcado $\sim$ aparqué

ast. SÉ QUE LU APARQUÉ II cast. he aparcado aparqué

ast. LU TUVE $\|$ cast. he tenido $\sim \underline{\text { tuve }}$

ast. DÓNDE COÑO LU APARQUÉ I| cast. he aparcado aparqué

ast. VINE II cast. he venido $\sim \underline{\text { vine }}$

ast. QUEDÉ D'ESTAR ALLÍ II cast. he quedado quedé

ast. QUE LO ANDUVE TODO II cast. he andado $\sim$ anduve

ast. DÓNDE COÑO LU DEJÉ II cast. he dejado $\sim \underline{\text { dejé }}$

ast. ÓNDE LU DEJÉ II cast. he dejado $\sim \underline{\text { dejé }}$

ast. TUVE QUE METELU II cast. he tenido $\sim$ tuve

Magar que nestos casos paez asumible qu'en castellanu l'usu previsible sería'l pretéritu perfectu compuestu, nun se pue refugar un usu tamién normal del pretéritu indefiníu simple nos mesmos contestos. En resultes, contemplamos la doble opción d’análisis. 
44. Tiempu simple y tiempu compuestu con 'haber': condicional simple y futuru imperfectu compuestu.

ast. NO TE LU LLEVARía II cast. habrá llevado

45. Comportamientu de la /-r/ final del infinitivu con un pronome enclíticu.

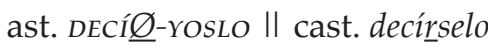

ast. PONE $\underline{\varnothing}_{M E}$ II cast. ponerme

ast. MET $\underline{\emptyset}_{L U} \|$ cast. meterlo

46. Unidá perifrástica verbal.

ast. NO SOI A DAR CON ÉL II cast. no puedo dar, no soy capaz de dar

47. Preposición en complexu verbal 'quedar' + infinitivu.

ast. QUEDÉ $\underline{D^{\prime} E S T A R}$ II cast. quedé en estar

48. Preposición 'a' en perífrasis verbal 'ir' + infinitivu.

ast. diba $\underline{\varnothing}$ recoyer I| cast. IBA $\underline{\text { A RECOGER }}$

49. Forma del lexema del sustantivu 'fíu -a' .

ast. $\underline{\underline{F} A}$ \| $\mathrm{cast} . \underline{\text { hija }}$

50. Forma del lexema del sustantivu 'muyer'.

ast. $\underline{\text { MUYER }}$ I| cast. $\underline{\text { mujer }}$

51. Forma del lexema del sustantivu 'llau'.

ast. llau I| cast. $\underline{L} A U$ (L्LADO)

Analizada más arriba la terminación ast. -au II cast. $-a(d)_{0}$ colos dos trazos distintivos ( $-d$ - intervocálica y marca de xéneru masculín - $u$ ), el pocu cuerpu fónicu d'esta palabra remite'l trazu lexemáticu a la diverxencia de formes qu' ofrez $l l$ - o $l$ - iniciales.

52. Forma del lexema del sustantivu 'imaxinación'.

ast. imaxinación || cast. IMAGINACIÓN

El cultismu imaxinación tien adaptación popular n'asturianu (por exemplu, recuéyese en Casu); hai tamién les formes imaxinar maxinar y maxín. 
53. Forma del lexema del sustantivu 'xulgáu'.

ast. $\underline{x u l g a ́ u ~} \sim \underline{x u z g a ́ u} \|$ cast. $\underline{\text { UZGAO }}$

54. Forma del lexema del sustantivu 'xoven' (o unidá léxica de sustantivu).

ast. $\underline{\text { mozu }}$ xoven $\|$ cast. LOVEN

Nesti casu, la BC tanto pue ser ente formes diverxentes d'un mesmu lexema orixinariu (ast. xoven II cast. joven) o ente dos lexemes diferentes (ast. mozu II cast. joven).

55. Forma del lexema de 'venti': elementu vocálicu nuclear.

ast. VENTE I| cast. veinte

ast. VENTE || cast. veinte

56. Forma del lexema de 'venti': terminación vocálica.

ast. venti I| cast. VENTE

ast. venti $\|$ cast. VENTE

57. Unidá léxica de sustantivu [BB] .

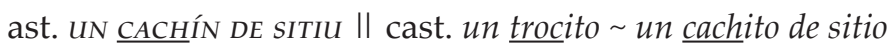

La BC ast. cachu II ast. trozo ye operativa na fala corriente. De toes maneres, en rexistros coloquiales del castellanu conozse tamién cacho col mesmu significáu que n'asturianu, polo que nesi casu sería una BCom.

58. Unidá léxica de sustantivu.

ast. UN $\underline{\text { RAPAZ }}$ I| cast. un $\underline{\text { muchacho }}$

ast. UN $\underline{\text { RAPAZ }}$ I| cast. un $\underline{\text { muchacho }}$

Na fala urbana d'Asturies hai un claru contraste ente ast. rapaz y cast. muchacho (chico).

59. Unidá sufixal de diminutivu [BB].

ast. UN CACHÍN II cast. un cach $\underline{i t o} \sim$ cachín

Xuégase equí con dos posiblidaes: una BC ast. -ín II cast. -ito; o una BCom ast. - in = cast. -ín, desque esti sufixu tien tamién dél usu nel castellanu norteñu.

60. Forma del lexema del verbu 'coyer'.

ast. coyía II cast. $\underline{\text { cogía }}$ 
61. Forma del lexema del verbu 'recoyer'.

ast. recoyer II cast. RECOGER

62. Forma del lexema del verbu 'alcontrar' [BB].

ast. alcuentro encuentro II cast. ENCUENTRO

63. Forma del lexema del verbu 'dir'.

ast. $\underline{\text { diría }}$ || cast. IRIÍA

ast. $\underline{\text { diba }} \|$ cast. $\underline{\underline{I}} B A$

64. Forma del lexema del verbu ' $\operatorname{tar}^{\prime}$.

ast. taba II cast. Y ESTABA UN RAPAZ

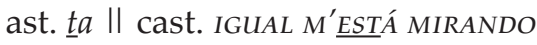

ast. taba \| cast. ESTABA MIRÁNDOLA

ast. taben II cast. ESTABEN ESPERANDO

ast. tar II cast. QUEDÉ D'ESTAR

65. Forma del lexema del verbu 'paecer'.

ast. paecía II cast. PARECÍA

66. Forma del lexema del verbu 'falar'.

ast. falo II cast. $\underline{\text { HABLO }}$

67. Forma del lexema del verbu 'dexar'.

ast. $\underline{\text { dexé }}$ II cast. $\underline{D E I E ́}$

ast. $\underline{\operatorname{dex}}$ II cast. $\underline{D E I O}$

ast. $\underline{\text { dexé }}$ II cast. $\underline{D E I E ́}$

68. Forma del lexema del tema de perfectu del verbu 'facer'.

ast. fici $\|$ cast. $\underline{\text { HICE }}$

69. Forma del lexema del verbu 'fixar'.

ast. fixate II cast. IIIATE

N'asturianu, la forma fixar, con /// y col significáu de 'fijar, asegurar, hincar', namái se recueye na fala tradicional en Balmonte de Miranda (DGLA, 2002-2004: s.v.). Ye posible que la forma reflexiva fixase (en) 'reparar en' seya un castellanismu, y qu'hubiere, en resultes, que clasificar 
esti ítem como un castellanismu léxicu con una BC ast. repara (en), date cuenta (de) II cast. fíjate (en). En tou casu, esta posibilidá de tipificación nun tendría repercusión nel cómputu, desque la forma rexistrada fijate clasifícase como castellana.

\section{Índiz d'asturianidá del testu de Xixón}

La llingua d'esti testu, y d'otros recoyíos nel mesmu trabayu, ta clasificada pola autora como «amestáu» (Prieto, 1991). Acordies colos criterios remanaos n'Andrés (2002), tipificaríemoslu como asturianu mínimu, pola presencia en cadena d'una serie de trazos estereotípicos o trazos-frontera de resistencia frente al castellanu: marca $-u$ de masculín singular, marca verbal de $3 .{ }^{\text {er }}$ persona del plural -en, pretéritu indefiníu simple, formes verbales sin marca -e de 3. ${ }^{\text {er }}$ persona (diz, vien), forma verbal ye, perda de $-r$ del infinitivu ante pronome clíticu, formes $-y$, $-y o s$ de pronome de complementu indirectu, sistema asturianu d'allugamientu de pronomes clíticos, sufixu diminutivu -ín y léxicu (rapaz, muyer, fía). Frente al testu de Cabranes, nun hai equí nengún otru trazu suplementariu del asturianu total.

Sometíu'l testu al procedimientu de cómputu propuestu, resulta:

(1) Corpus sintéticu d'algame estrechu (CSAE)

Bases contrastives del corpus: 54

Términos contrastivos asturianos: 21,7

Índiz d'asturianidá testual: 4,01

(2) Corpus sintéticu d'algame ampliu (CSAA)

Bases contrastives del corpus: 69

Términos contrastivos asturianos: 19,70

Índiz d'asturianidá testual: 4,30

(3) Corpus analíticu d'algame estrechu (CAAE)

Bases contrastives del corpus: 130

Términos contrastivos asturianos: 44 
Índiz d'asturianidá testual: 3,38

(4) Corpus analíticu d'algame ampliu (CAAA)

Bases contrastives del corpus: 145

Términos contrastivos asturianos: 65

Índiz d'asturianidá testual: 4,48

(5) Índiz mediu d'asturianidá testual: 4,04

\section{Conclusiones}

El siguiente gráficu muestra comparativamente los índices d'asturianidá rexistraos en testu de Cabranes y en testu de Xixón:

A la vista del gráficu, sáquense les siguientes conclusiones:

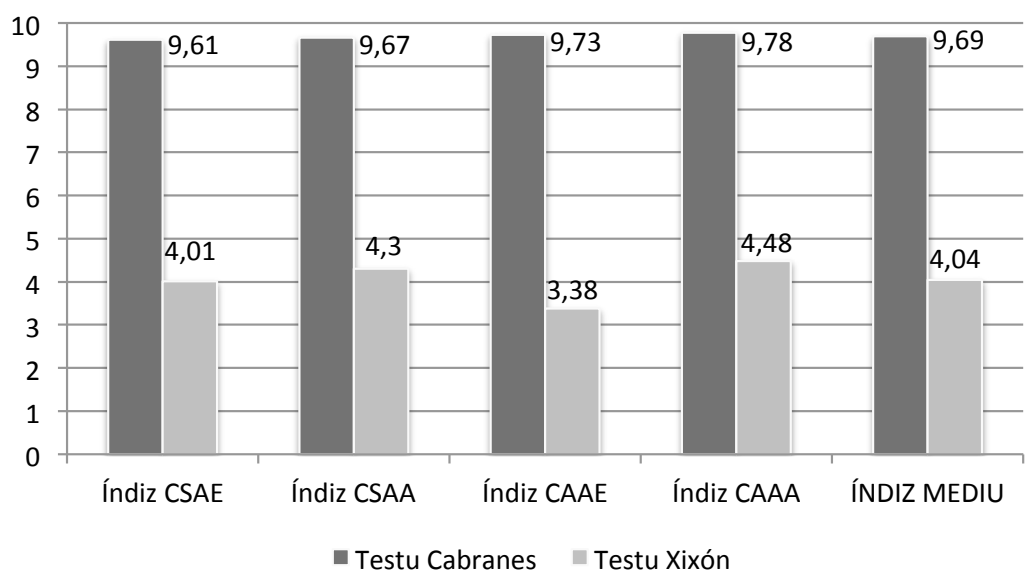

-L'ensayu colos cuatro corpus contrastivos ofrez unes diferencies bultables ente dambos testos: el de Cabranes supera siempre 9,50, y el de Xixón nun algama nunca 4,50. L'índiz mediu d'asturianidá de Cabranes ye de 9,69, frente al 4,04 de Xixón.

-Como se ve, el valor mediu algamáu pol testu de Cabranes ye mui altu y cercanu a la saturación. Representa la llingua d'una falante rural d'un conceyu de perpoca influencia urbana nos años 
40 del sieglu XX. El testu analizáu amuestra un idiomatismu asturianu enforma eleváu, prueba del altu grau de conservación de la llingua autóctona. Nun hai duda de que'1 testu de Cabranes ta en llingua asturiana.

-Pela cueta, l'índiz mediu d'asturianidá del testu de Xixón ye un valor mui pembaxo del testu cabraniegu. Como yá dixemos, nuna primer güeyada, y ensin nengún análisis cuantitativu, nun tendríemos inconveniente en tipificar el testu como amestáu mínimu, pola presencia d'una serie de trazos-frontera estereotípicos y pola ausencia simultánea d'otros trazos que cayeríen nel asturianu total. Un observador profanu, y mesmamente un llingüista, afirmaría con seguranza que'1 testu nun ta en castellanu, sinón n'asturianu urbano. Sin embargu, d'acuerdu col procedimientu equí propuestu, el testu de Xixón amuestra cifres claramente pembaxo del puntu mediu de 5, que marcaría la llende ente' 1 «campu» del castellanu y el del asturianu. En términos escolares, podríemos dicir que'1 testu de Xixón «nun aprueba l'esame d'asturianu», desque estadísticamente paez axuntar una proporción mayor de trazos castellanos que d'asturianos. Podría dicise, entós, que'1 testu de Xixón ye más castellanu qu'asturianu; o que ta en castellanu con dellos trazos asturianos ${ }^{21}$.

Dende'l puntu de vista del procedimientu, podríen sacase estes otres conclusiones:

-Les diferencies ente dos llingües asemeyaes, como asturianu y castellanu, son cuantificables y midibles. La unidá de midida yél fenómenu contrastivu y los trazos diferenciales en que se manifiesta.

-Nun testu llingüísticu híbridu ye posible determinar el pesu d'una de les llingües de dos maneres: o bien de manera percepti$v a$, atribuyendo a una serie de trazos el valor delimitativu que

21 Hai qu'entender esta afirmación dende'l puntu de vista del métodu remanáu equí pa estos dos testos concretos. Necesitábase contrastar el pesu de los trazos morfosintácticos (gramaticales) sobre'l restu, pa ver qué sentíu tien l'afirmación de qu'«un testu ta n’asturianu si tien gramática asturiana». 
supuestamente los falantes-yos dan; o bien de manera oxetiva, detectando los trazos diferenciales, tipificándolos na so adscripción llingüística, y calculando la proporción de trazos d'una llingua y de la otra realizaos efectivamente en testu. Esto segundo ye lo que fixemos nesti ensayu.

-El procedimientu ensayáu demuestra que ye posible cuantificar y midir el grau o proporción qu'algamen los trazos d'una y otra llingua nun testu determináu. Pa ello ye imprescindible establecer les bases contrastives y los términos contrastivos, pa depués computar les escoyetes de caúna de les dos llingües.

-En resultes, un retu pal estudiu de los lectos híbridos ye estremar ente los resultaos d'un enfoque suxetivu (perceptivu) del falante y los d'un enfoque oxetivu (cuantitativu) del llingüista. La entruga de les encuestes sociollingüístiques, del tipu «¿qué ye lo que fala usté?» o asemeyaes, llogra respuestes de tipu perceptivu, que nun tienen por qué coincidir con un análisis oxetivu per parte del llingüista. Esta incoincidencia enseña dos verdaes que puen coesistir, siempre que caúna xuegue en terrén distintu: una na sociollingüística y otra na llingüística.

\section{Referencies bibliográfiques}

Alba Niño, M. (2011) "Fronteras lingüísticas en el ciberespacio", en Andrés Díaz, R. de (coord.), Lengua, ciencia y fronteras, Uviéu, Ediciones Trabe / Universidá d’Uviéu, 213-244.

Andrés, R. D' (1993) Allugamientu de los pronomes átonos col verbu n'asturianu, Uviéu, Universidá d’Uviéu.

Andrés, R. D' (2002) “L'asturianu mínimu urbanu. Delles hipótesis", Lletres Asturianes, 81, 21-38.

ANDRÉs, R. D' (2011) “Fronteras lingüísticas y geotipos, con atención a la zona Eo-Navia", en Andrés Díaz, R. de (coord.), Lengua, ciencia y fronteras, Uviéu, Ediciones Trabe / Universidá d'Uviéu-Seminariu de Filoloxía Asturiana, 121-152. 
Andrés, R. D’ (2015) “Un esbozu pal estudiu de les interferencies y ultracorrecciones fóniques ente l'asturianu y el castellanu", en Andrés, R. d'; Fernández Lorences, T. \& Villaverde Amieva, J. C., Varia asturlleonesa n'homenaxe a José A. Martínez, Uviéu: Ediciones Trabe / Universidá d'Uviéu, 137-163.

Andrés DíAz, R., Álvarez-Balbuena García, F., Cueto Fernández, M. y Suárez Fernández, X. M. (2013) "About the concept of "geodifferential feature" between linguistic varieties in contact", en Carrilho, E.; Magro, C. \& Álvarez, X. (edd.), Current Approaches to Limits and Areas in Dialectology, Cambridge, Cambridge Scholars Publishing, 85-122.

Canellada, M. J. (1944) El habla de Cabranes, Madrid, Revista de Filología Española, anejo XXXI.

Cano González, A. M., Conde Saiz, M. V., García Arias, J. L. y García González, F. (1976) Gramática bable, Madrid, Ed. Naranco.

Cuesta, G. (2016) “Identidad lingüística y fenómenos de transferencia en La Pola de Lena (Asturias)", Lletres Asturianes, $115,147-172$.

DGLA = García ArIAs, X. Ll. (2002-2004) Diccionario general de la lengua asturiana, Oviedo, Editorial Prensa Asturiana / La Nueva España. Online: <mas.lne.es/diccionario/>.

DRAE $=$ Real Academia Española, Diccionario de la lengua española. Online: <http://dle.rae.es/?id=DgIqVCc $>$; consultes: 13 de xunu del 2017.

Dyzmann, N. V. (2000) "Averamientu al estudiu del contautu de llingües (asturianu-castellán) nel Principáu d'Asturies", en Lletres Asturianes, 73, 93-105.

Elizaincín, A. (1996) “Los estudios lingüísticos en la frontera uruguayobrasileña", en Carrasco González, J. M. y Viudas Camarasa, A. (eds.) Actas del Congreso Internacional Luso-Español de Lengua y Cultura en la Frontera (Cáceres, 1 al 3 de diciembre de 1994), vol. I, Cáceres, Universidad de Extremadura, 267-275. 
Fernández Álvarez, F. J. (1989) “Influjo del castellano en Llue (Colunga)", Lletres Asturianes, 33, 35-52.

Fernández Lorences, T. (2011) "Alternancia y mestura de códigu asturianu / español. Problemes nel determín de les fronteres llingüístiques", en Andrés Díaz, R. de (coord.) Lengua, ciencia y fronteras, Uviéu, Ediciones Trabe / Universidá d'Uviéu, 191-212.

GLA = Academia de la Llingua Asturiana (2001) Gramática de la llingua asturiana (3ª ed.), Uviéu, Academia de la Llingua Asturiana.

GLHA = García Arias, X. LL. (2003) Gramática histórica de la lengua asturiana, Uviéu, Academia de la Llingua Asturiana.

GPA = ANDRÉs, R. D' (1999) Gramática práctica de asturiano (3a ed.), Mieres, Editora del Norte.

Hernández Alonso, C. (1999) "Castilla la Vieja”, en Alvar, M. (dir.) Manual de dialectología hispánica. El español de España, Barcelona, Ariel, 197-212.

Kabatek, J. (1997) “Dime cómo hablas y te diré quién eres. Mezcla de lenguas y posicionamiento social", Revista de Antropología Social, 6, 215-236.

López Morales, H. (2010) Sociolingüística (3ª ed.), Madrid, Gredos.

LÜDTKE, J. (1999) “Las variedades contactuales y el asturiano", Lletres Asturianes, 72, 23-44.

Martínez Álvarez, J. (1968) Bable y castellano en el concejo de Oviedo, Oviedo, Archivum (XVII).

Prieto, C. (1991) Investigación sociollingüística na Plaza'l Sur de Xixón, Uviéu, Serviciu de Publicaciones del Principáu d'Asturies.

Teso, E. del (2015) “L'amestáu urbanu. Cuestiones de normativa y llingua escrita”, Lletres Asturianes, 113, 11-26. 
Viejo Fernández. X. (2005) “El contacto de castellano y asturiano en Asturias»" en Ferrero, C. y Lasso-Von Lang, N. (eds.), Variedades lingüísticas y lenguas en contacto en el mundo de habla hispana, Bloomington, Author House ed., 138-145.

RAMÓN D'ANDRÉS UNIVERSIDÁ DE UVIÉU randresd@uniovi.es 
